\title{
CIENCIA Y TECNOLOGÍA, EDUCACIÓN Y CIUDADANÍA
}

\author{
Oscar Alamo* \\ Lisha DÁVILA**
}

Recebido: 22 nov. 2010

Aprovado: 09 fev. 2011

* Docente-Investigador. Instituto de Ciencias Sociales - Universidad Nacional de Villa María. Córdoba. Argentina. Email: oalamo@elsitio.net

** Licenciada en Comunicación Social. Miembro do Programa de Escritura, difusión y publicaciones científicas. Centro de Estudios Avanzados - Universidad Nacional de Córdoba. Córdoba, Argentina. Email: lishpam@gmail.com

Resumen: La convergencia entre Ciencia, Educación y Ciudadanía, proponen una diversidad disciplinar que, en no pocas oportunidades, convergen en un cúmulo fragmentado de buenas intenciones intelectuales. En particular, pensar algunos aspectos en cuanto al desarrollo social y distribución del conocimiento, como elemento dinámico que induzca a la relación entre estas tres grandes categorías, implicaría una particular visión al momento de articular definiciones que permitan identificar líneas (generales) con el objeto de instrumentar y gestionar políticas públicas en ciencia y tecnología (CyT). En tal sentido, el presente trabajo propone abordar desde una perspectiva crítica, como se articulan éstas categorías y su incidencia en relación con la percepción de los avances científicos e innovación tecnológica, por parte de los ciudadanos, considerados éstos como sujetos políticos. Principalmente, a partir de la convergencia de cuestiones que tienen que ver con conceptos tales como: democratización del conocimiento, incertidumbre y riesgo, y la participación de la Universidad en su dimensión social. Ésta última, como institución en la cual se genera la mayor parte de ciencia en América Latina.

Palabras claves: Ciudadanía. Educación. Universidad. Riesgo. Ciencia y Tecnología.

\section{SCIENCE AND TECHNOLOGY, EDUCATION AND CITIZENSHIP}

Abstract: The convergence between Science, Education and Citizenship proposes a disciplinary diversity that, in not few opportunities, lead to a fragmented heap of good intellectual intentions. Especially, to think some aspects as for the social development and distribution of the knowledge, as dynamic element that it induces the relation between these three large categories, it would imply a particular vision to the moment to articulate definitions that allow to identify (general) lines in order to orchestrate and to manage public policies in science and technology (C\&T). To this respect, the present work proposes to approach from a critical perspective, since these articulate categories and his incident in relation with the perception of the scientific advances and technological innovation, on the part of the citizens, considered these as political subjects. Principally, from the convergence of questions that they have to see with such concepts as: democratization of the knowledge, uncertainty and risk, and the participation of the University in its social dimension. The latter, as the institution in which most of science is generated in Latin America.

Key words: Citizenship. Education. University. Risk. Science and Technology.

\section{INTRODUCCIÓN}

Al analizar, desde una perspectiva macro, la relación entre Ciencia, Educación y Desarrollo Social, se presentan un amplio espectro de categorías analíticas al momento de explicar los distintos fenómenos sociales que éstas involucran. 
Un breve análisis retrospectivo, muestra que la asimilación de determinados paradigmas en términos de producción y generación de conocimiento en CyT, principalmente los propuestos desde los países centrales, son impuestos como consecuencia de una dinámica estructura geopolítica. Tal como aconteciera en el auge desarrollista, se intenta seguir o imitar a los países desarrollados, para alcanzar un estadio superior revestido de cierto progreso lineal y como consecuencia de un modelo único (hegemónico). "En este contexto, los problemas de instrumentar políticas en CyT, son sólo tácticos, pues la estrategia viene dada desde afuera y solo trata de "cerrar brechas"” (VARSAVSKY, 1973, p. 59).

Una problemática emergente, viene dada al estudiar la percepción de los avances científicos y la innovación tecnológica, por parte de los ciudadanos en un marco de complejas transformaciones. Por lo tanto, resulta imprescindible enfocar y cuestionar el alcance de estas mudanzas. ¿Se promueve la participación ciudadana en relación a una aproximación sobre CyT ?, ¿ Es ejercido el derecho de ciudadanía en la formulación de políticas públicas en CyT ?. Interrogantes mínimos que debieran ser considerados frente a diversas situaciones y contextos propuestas por una cotidianidad científico-tecnológica, en general poco visible.

\section{DESARROLLO}

En una primera aproximación, es menester identificar que la creciente evolución y producción de conocimiento en $\mathrm{CyT}$, en el marco de las denominadas sociedades occidentales, se expande dinámicamente hacia nuevos horizontes y en su correlato alcanza nuevas modalidades de gestión y organización social. En tal sentido, la emergencia de complejos paradigmas conlleva a nuevas prácticas para la apropiación del conocimiento, las cuales son significadas en relación directa con las capacidades ${ }^{1}$ propias de cada y determinado espacio social.

En general y de acuerdo a los modelos tradicionales de enseñanza, las verdades científicas son expuestas como un saber universal e independiente de la realidad, sin tener en cuenta las distintas prácticas sociales que pudieran contener o referir, incluso a la apropiación de una determinada tecnología.

Para una gran mayoría, los conocimientos científicos son incuestionables, fuente única de validación de la verdad. Es decir, conocimiento cierto y objetivo

1 La formalización del concepto de diferenciación de capacidades tecnológicas, se enmarca principalmente en el estudio de la economía del cambio tecnológico. No obstante, se debe tener en cuenta que la centralidad ocupada por las estrategias de formación e innovación en los países desarrollados, no implican de manera alguna que éste sea el modelo a seguir por los países en vía de desarrollo. 
sobre de la realidad. Así, la enseñanza consistía cada vez más en la transmisión de resultados, de conceptos y de doctrinas, y en el mejor de los casos, de métodos que se inculcaban a los alumnos, sin tener en cuenta las circunstancias que habían presidido su elaboración. "El estilo de enseñanza científica no era para nada narrativo -es decir, inmerso en significaciones humanas-, sino sobre todo dogmático, es decir que presentaba verdades poco contextualizadas" (FOUREZ, 1994a, p. 34).

Se ha podido observar, en casi todos los niveles de enseñanza, que las verdades científicas son presentadas como un saber desanclado. En consecuencia, bajo ésta concepción de impartir saberes, los mismos se convierten en cuestiones objetivas, deslocalizadas y ajenas -en términos de pertenencia social- a los intereses de los educandos, y por ende, de la comunidad a la cual pertenecen.

Dotar de contenido social-contextual, posibilita el desarrollo de una concepción sobre las relaciones entre ciencia, tecnología y sociedad diferentes de las actualmente diseminadas, predominantes en los ámbitos formales de producción de conocimiento.

[...] La adaptación de nuestros modos de conocer a las estructuras de las ciencias modernas no se percibe como algo optativo; por el contrario, se presenta como una necesidad si se quiere conservar el status social. Nos podemos felicitar por los beneficios que proviene de ese espíritu científico, pero sería una equivocación no darse cuenta de que lo que se pide es adaptarse a un modo particular de conocer impuesto por el sistema de los saberes científicos y abandonar otras formas de saber (FOUREZ, 1994b, p. 157).

Desde un enfoque CTS, es posible platear un abordaje alternativo, crítico a los métodos tradicionales en cuanto a la enseñanza de la ciencia. En tal sentido, se consideran la posibilidad de incorporar cambios al momento de considerar y valorar los diferentes aspectos relacionados con el conocimiento y prácticas de los distintos actores involucrados en el proceso de enseñanzaaprendizaje.

Según López Cerezo (2002), en la actualidad los estudios CTS se constituyen por una diversidad de programas de colaboración multidisciplinar que, enfatizando la dimensión social de la ciencia y la tecnología, comparten: “a) El rechazo de la imagen de la ciencia y la tecnología como una actividad pura; b) la crítica sobre la concepción de la tecnología como ciencia aplicada y neutra; c) condena de la denomina tecnocracia".

La integración que propone éste enfoque, en relación con la enseñanza de la ciencia, en general, apunta a conformar estructuras formales e informales, que 
fomenten una educación más cercana a valores humanistas, que como espacio de llegada contemple todo un ámbito social determinado, con el objeto de evitar la fragmentación. Inserta en su contexto de pertenencia, tal situación, posibilita mejorar la actitud y el interés de los educandos en relación a la ciencia y a su aprendizaje.

El aprendizaje de conceptos y procedimientos científicos en ámbitos propios, suministra condiciones necesarias (no suficientes) para los individuos, a la vez que posibilita una mayor adaptación crítica, al tiempo que contribuye a superar las restricciones impuestas por los contextos físicos y culturales.

Al utilizar elementos de la realidad social de los educandos en los ambientes de enseñanza, no solamente se facilita el aprendizaje de conceptos, sino que también se desarrolla dentro del proceso de enseñanza-aprendizaje la actitud de asociar los conocimientos científicos con la realidad vivenciada.

Por consiguiente, al reconocer, reelaborar y difundir saberes de una comunidad, se establecen conocimientos que fortalecen determinada identidad cultural. De esta forman, una institución orientada a la formación, en su carácter de locus privilegiado de la enseñanza de conocimientos científicos, aumenta su legitimidad social y vínculos con la comunidad.

Es claro que si bien la ciencia se encuentra presente a diario en la vida de los seres humanos, en escasas oportunidades es presentada -particularmente en la enseñanza básica-, la relación que existe entre ciencia, tecnología y sociedad. Una sociedad usufructúa los producidos de la ciencia sin comprender su naturaleza, sus ventajas y desventajas, sus límites y sus riegos. Es en este contexto que el proceso de enseñanza de la ciencia, desde una perspectiva CTS, puede contribuir a la formación de individuos capaces de opinar acerca de los destinos de la ciencia y la tecnología, ejerciendo su ciudadanía al respecto.

Avanzar en esta línea implica adoptar posiciones que nos remiten al concepto de "democratización del conocimiento", comprendido éste, en su dimensión política asociada a la actividad de enseñar ciencia y a la alfabetización en Ciencia y Tecnología. Se trata de una opción conceptual e ideológica, que tiene fuertes correlatos a nivel instrumental, sobre la forma de enseñar (RIETTI, 1999).

\section{SOBRE RIESGO E INCERTIDUMBRE}

El vertiginoso desarrollo tecnológico está domesticando (en sentido Faústico) la naturaleza. El poder del átomo y la potencialidad de la información genética proponen un nuevo mundo. Un mundo inimaginable, impensado, hasta cada una de éstas innovaciones, pero también, un mundo en el que la 
sensación de amenazas crecientes, son consecuencia de los peligros creados por ese desarrollo.

En el "mundo del riesgo" o la denominada "Sociedad del Riesgo", asociada a la ciencia y la tecnología actual: "cuanto más conocemos los riesgos, mejor apreciamos la gran extensión de nuestra ignorancia; cuanto más hacemos por controlarlos, mayores son los riesgos generados en otra parte del sistema" (LÓPEZ CEREZO; LUJÁN LOPEZ, 2000, p. 86).

Disponer de una sociedad con capacidad de opinión en relación a los aspectos que acompañan y operan sobre las políticas públicas en CyT, son consecuentes con la necesidad imperiosa al momento de analizar los desarrollos científicos tecnológicos en su conjunto. Es decir, no solamente objetivar sobre las bondades de la innovación, sino también, observar los niveles de incertidumbre y los riegos potenciales en la aceptación de una innovación tecnológica determinada.

Una alternativa consiste en estimular el alance e interés social para obtener una discusión, una agenda, una posibilidad de consenso sobre las implicancias de la adopción y/o desarrollo en ciencia y tecnología. Se trata entonces de promover la participación en la discusión directa sobre aquellos temas que llevan a la formulación de políticas públicas en el área de ciencia y tecnología.

En tal sentido, acercar a los ciudadanos a las actividades y/o disciplinas científicas, no tendrá como propósito alcanzar conocimientos específicos, nada que implique lo que significa hacer ciencia en un laboratorio -tareas propias-, más bien tiene que ver con discusiones sobre resultados y tendencias, en definitiva con proveer mejor calidad de información para la toma de decisiones.

Ahora bien, el concepto de sociedad del riesgo, sin embargo, llama la atención sobre el limitado control de los peligros como consecuencia de la arrolladora influencia del cambio científico-tecnológico. "Y la principal cuestión es cómo tomar decisiones en condiciones de incertidumbre fabricada, cuando no sólo es incompleta la base de conocimiento, sino que disponer de más y mejor conocimiento frecuentemente supone más incertidumbre" (BECK, 2002, p. 19).

Históricamente se ha podido observar que la mayor participación de ciudadanos en discusiones relacionadas con actividades científico-tecnológicas, puntualmente, han venido de la mano de las denominadas grandes innovaciones. Tal situación aconteció, por nombrar algunas, con la energía nuclear, el genoma humano, los organismos genéticamente modificados, las células madres, la manipulación de la materia en dimensiones nanométricas, entre otras. Estas discusiones sólo se han dado en determinados niveles sociales, la

2 Concepto desarrollado originalmente por el sociólogo alemán Ulrick Beck. Quien en 1986 enuncia que la tecnología actual ha creado nuevas formas de riesgos e impone una peligrosidad cualitativamente distinta. 
información difundida no siempre ha sido calificada y menos aún provista en las dimensiones necesarias.

El condicionamiento que conlleva la adopción de determinada tecnología indicaría nuevas formas de existencia individual y social.

Si un país adopta la electricidad nuclear, se hará ver que hay que adoptar policía y sistemas de seguridad y de protección para evitar sabotajes. Ante eso, el planteamiento general que pretende separar las tecnologías de sus aplicaciones parece inconsistente porque las situaciones concretas muestran que el modo de vida se adapta a la tecnología (FOUREZ, 1994b, p. 156).

Por lo general, existe entorno a una innovación tecnológica una tendencia a ser presentada (vendida), como algo positivo (como algo ventajoso). No obstante, no se acompaña información (menos aún campañas, consultas, foros) sobre los riegos potenciales (o reales) de la apropiación y/o aplicación. Tampoco, sobre el nivel de incertidumbre que acompaña a todo desarrollo científico-tecnológico.

Una toma de posición sobre éstos aspectos permite, al menos pedir cuentas a los encargados en la formulación de políticas y la gestión de las mismas, ya que tanto riesgos como peligros son atribuidos a daños inciertos. Si son vistos como fortuitos o contratiempos, serán entendidos socialmente como peligrosos; pero si se perciben como fruto de decisiones, serán entendidos como riegos que conllevan imputabilidad (LÓPEZ CEREZO, 2002, p. 55).

Por su parte, los conceptos científicos de riesgo e incertidumbre no dependen únicamente del escrutinio científico de una realidad objetiva y compleja, sino que son asimismo el resultado de valores e intereses humanos que forman una parte inherente de la ciencia.

La carencia o escasez de información produce un alejamiento, -por parte de los distintos actores sociales-, de las actividades relacionadas con desarrollo científico y tecnológico. Justamente en un momento en el cual las nuevas tecnologías alcanzan un alto nivel de impacto en la cotidianidad de nuestras sociedades.

Digamos que ésta cotidianidad ha incorporado -de manera bastante implícita- el concepto de riesgo, el cual se ha convertido en algo con lo que se debe convivir. La presentación del riesgo en una modalidad difusa, desdibujada en sus límites y alcances, tiene como corolario su falta de identificación explícita al momento de ser considerado como objeto de reflexión. Luego, es naturalizado. 
Por lo tanto, el crecimiento en la obtención de innovaciones tecnológicas, sustentado por el modelo tecno-mercantil imperante, implica el crecimiento en la probabilidad de la producción de daños en cualquiera de sus modalidades, ya sean como daños súbitos o subyacentes. Los mismos se consideran asociados a la universalidad de la tecnología y sus consecuencias negativas -daños colaterales o secuelas no deseadas-, no distinguirían entre barreras nacionales, clases sociales o generacionales.

Presentada en estos términos, ésta problemática conduce a inferir que afectaría a todas las sociedades por igual, sin distinción alguna; y que cierta aleatoriedad presidiría esta línea de pensamiento.

Pero es menester dejar por sentado, que también existen marcadas inequidades en cuanto a la distribución del riesgo. La actual necesidad de su distribución está lejos de reemplazar a la problemática de la distribución de la riqueza, no obstante ser esta última un factor condicionante para el abordaje de las emergencias a que conlleva (el riesgo) en muchos lugares y para numerosos colectivos sociales.

El carácter social de incorporar innovaciones tecnológicas, estaría indicando que aparte de la formación para alcanzar una mejor calidad de ciudadanía, es también necesario el abordaje multidisciplinar para este tipo de cuestiones. Pero aún así, no alcanza solamente con la perspectiva de los científicos, es necesario también contar con la opinión de los organismos gubernamentales en CyT, las empresas y, fundamentalmente, considerar la opinión de la ciudadanía en su conjunto.

\section{CIENCIA, TECNOLOGÍA Y UNIVERSIDAD}

El conocimiento científico y tecnológico, y su extensión a escala global, han dado cabida a nuevas formas de enfocar (segmentar) a la sociedad, surgen la sociedad del riesgo, la sociedad de la información, luego sociedad del conocimiento. Concepciones que si bien hacen su aporte en términos de un amplio surtido de categorías analíticas, ponen en evidencia una visión fragmentada.

Prósperas en ambigüedades, consensuales a bajo costo, estas semánticas emergentes, acarrean el defecto de sortear una cuestión central: la pluralidad de los conocimientos y de sus protagonistas.

Desde los estudios en CTS, uno de los aportes más significativos, se constituye a partir de la ruptura epistemológica de las últimas décadas. Dotan de significado a un nuevo modelo de "revalorización del sujeto", tanto en las ciencias humanas, como en las ciencias sociales. 
Se trata precisamente de la rehabilitación de los conocimientos procedentes de las experiencias vividas. Los conocimientos fundamentales o ilustrados, los conocimientos aplicados de los expertos y los contra-expertos, los conocimientos ordinarios que asoman en la cotidianidad de las diferentes prácticas sociales.

Tradicionalmente la innovación tecnológica ha implicado una relación vertical y unilateral con las poblaciones a las que se dirige. Los paradigmas emergentes identificados con las línea de estudios CTS, no han logrado concretar hasta el momento, un avance significativo en relación con la instrumentación de políticas públicas en CyT que modifiquen tal situación.

La historia reciente demuestra que, particularmente en el la relación conocimiento-trabajo, se acentúa la reproducción de un sistema de autoridad y la división internacional del trabajo. En tal sentido, la innovación científica y tecnológica, ante la emergencia de su dimensión socio-cultural, se constituye en un factor significativo para la construcción de las sociedades contemporáneas.

Observado lo enunciado, se puede acordar en el marco de nuestras sociedades, que el conocimiento científico y tecnológico, opera como dispositivo de transformación, razón por la cual se constituye en un factor significativo incorporado en la cotidianidad de distintas prácticas sociales. Es en tal sentido, que cabe requerir la atención de aquellos actores involucrados en fomentar la difusión de actividades y el desarrollo de la ciencia y la tecnología.

Por su parte, es del Estado la responsabilidad de impulsar a la educación como herramienta de igualdad y democracia. En particular, son las Universidades -y sobretodo en aquellas de origen estatal- los organismos educativos responsables de la mayor parte de la investigación académica que se hace en países de Latinoamérica.

Una tendencia de los últimos años, indica que en general la relación entre Universidad y Sociedad, estaría orientada principalmente, en dirección a satisfacer las necesidades productivas de determinado sector de la sociedad civil. Por ende, los problemas emergentes asociados a los modos de estrechar vínculos entre investigación científica, innovación tecnológica y la sociedad civil, tienden a acentuar diferencias estructurales y conceptuales.

Conceptos como investigación, ciencia, tecnología, innovación, calidad y transferencia, son abordados desde una perspectiva dinámica, asociada en general, con determinada lógica productivista, formulada principalmente desde algunos de los escenarios que imponen el mercado global.

Es muy común en nuestro medio hablar de la relación universidad-empresa, gestora, incubadora, entre otras potencialidades, siempre teniendo la idea de 
que es la empresa la que puede hacer que el conocimiento que se genera en la universidad pueda servir a la sociedad.

Para el caso de América Latina, se otorga un papel decisivo al desempeño de las universidades y los organismos autárquicos de investigación dependientes de los estados nacionales. Si bien es menester, reconocer que en la actualidad existe un clima de opinión acerca de que éstas instituciones representan un locus privilegiado donde deben crecer la investigación, la transferencia y la innovación (sistemas de I+D organizado en torno a disciplinas), articulándose con el otro locus obligado: los laboratorios industriales (organizados por el tipo de producto final).

Sin embargo, Renato Dagnino afirma que es posible observar que las Universidades públicas en Latinoamérica revisten un carácter disfuncional para con la sociedad que la contiene, para su contexto económico, político y social. No se sabría bien para que, o no se tendría en claro hacia quienes se encuentra destinada su gestión. "Me atrevo a decir que la Universidad perdió su funcionalidad y no sirve a la clase dominada, ni a la clase dominante" (DAGNINO, 2007). Esta última, importa conocimiento, independientemente de lo que pase en los países desarrollados, donde la universidad produce conocimiento que interesa a la empresa. Para la clase dominada -trabajadora-, hace mucho tiempo que la Universidad dejó de ser una vía de ascenso social y el conocimiento producido no es de relevante para la clase dominada.

Es entonces, en el espacio de la formulación (convergencia) de políticas para la vinculación, donde se instala la necesidad de propiciar nuevas formas de asociación entre Universidad y organizaciones socio-productivas, sin descuidar las prioridades, o los lineamientos centrales, en términos del contenido social de las políticas públicas en CyT. Estableciendo a su vez, eficientes mecanismos institucionales de cooperación, procesos interactivos y nuevos mapas de colaboración en la definición de estrategias.

Otra cuestión, no menos importante y para tener en cuenta, radica en que para el caso de ausencia o deficiencia de los actores de la industria en los procesos de transferencia e innovación, suele trasladarse casi automáticamente la responsabilidad a las universidades, como ha ocurrido y ocurre en los países denominados periféricos.

Desde los sectores socio-productivos, se espera que estas instituciones se adapten, que salgan a buscar patrocinadores o inversiones de riesgo, que destinen recursos a reconvertir sus líneas de trabajo, que exploren el mercado en busca de clientes que requieran su expertise. 
Si se trata de incentivar la articulación entre academia e industria, se espera que la primera invierta, mientras se ofrecen facilidades y estímulos de diverso tipo a la segunda. Por último, y como consecuencia de las dos primeras operaciones, pensar el papel de la universidad en la nueva sociedad del conocimiento implica concentrarse en analizar a la primera para ver de qué manera responde a las anteriores demandas (VARA et al, 2007).

Si bien numerosos autores acuerdan en la necesidad de alcanzar compromisos sociales por parte de los actores identificados con el campo de la educación y la divulgación científica; no menos importante es la consolidación de un modelo participativo, que alcance a las comunidades científicas locales, con identidad e intereses propios y menos susceptibles a las mudanzas científico-tecnológicas importadas de los países centrales.

En general en nuestras universidades se sigue bajo un modelo de enseñanzaaprendizaje autoritario, que es heredado del siglo pasado, ineficaz, y sobre todo, el tema de la neutralidad y el determinismo.

Por lo tanto, levantar la cuestión del mejoramiento de la enseñanza de la ciencia se inscribe en una antigua tradición educativa en el país y la universidad, dirigida a democratizar el conocimiento. [...] "Entendiendo que estos aspectos representan una responsabilidad que emana del propio proceso de investigación; y que por lo tanto, deben constituirse desde el origen en uno de los ejes de una política para la ciencia" (RIETTI, 1999).

Por lo tanto, la producción de conocimiento científico en Latinoamérica debe ser concebida a partir de una comprometida política de vinculación con su enseñanza y democratización. Con el fin de consolidar un modelo inclusión social como necesaria consecuencia de la relación que emerja entre Ciencia, Universidad y Sociedad.

[...] la capacidad crítica de los científicos y los tecnólogos jóvenes, puede ser decisiva para encontrar nuevos caminos.

Para liberar esta fuente potencial de creación, es necesario cambiar algunos viejos hábitos de la comunidad científica, entre ellos, el peso que se le asigna habitualmente a la experiencia en la asignación de tareas, especialmente en el terreno de la tecnología. No cabe duda que la gran importancia que se le da a la experiencia tiene su justificación en situaciones "convencionales". En lo que estamos tratando, sin embargo, es indispensable romper con los criterios convencionales, y afrontar los riesgos que ello significa (HERRERA, 1973, p. 18). 


\section{REFERENCIAS}

BECK, Ulrich. La sociedad del riesgo global. España: Siglo XXI, 2002.

DAGNINO, Renato P. Neutralidade da ciencia e determinismo tecnológico. Campinas: UNICAMP, 2008.

. Empezando por la extensión universitaria. Conferencia

presentada en II Seminario Iberoamericano de Ciencia y Tecnología para el Hábitat Popular. Córdoba, Argentina, 2007.

FOUREZ, Gérard. Alfabetización científica y tecnológica. Buenos Aires: Ediciones Coihue, 1994a.

La construcción del conocimiento científico. Sociología y ética de la ciencia. Madrid: Editorial Narcea, 1994b.

HERRERA, Amilcar. La creación tecnológica como expresión cultural. Nueva Sociedad: Buenos Aires, n. 8-9, p. 58-70, sept./dic., 1973.

LÓPEZ CEREZO, José A.; LUJÁN LÓPEZ, José L. Ciencia y política de riesgo. Madrid: Alianza Editorial, 2000.

LÓPEZ CEREZO, José A. Ciencia, tecnología y sociedad: el desafío de la interacción. Londrina: IAPAR, 2002.

RIETTI, Sara. Políticas de ciencia, tecnología y educación para la democratización del Conocimiento. La perspectiva desde una política para la ciencia y el desarrollo educativo. Buenos Aires, sept./oct., 1999. Disponible en: <http://www.oei.es/salactsi/sara3.htm>. Acedido en: 15 sept. 2010.

RIETTI, Sara; MASSARINI, Alicia. Democratización del conocimiento. Diccionario del pensamiento social alternativo. Buenos Aires: Editorial Gedisa, 2006.

VARA, Ana M.; MALLO, E., HURTADO, D. Universidad y sociedad del conocimiento: apuntes históricos y perspectivas actuales en el contrapunto entre centro y periferia. Centro de Estudios de Historia de la Ciencia y la Técnica José Babini. Buenos Aires: UNSAM, 2007.

VARSAVSKY, Oscar. Hacia una política científica nacional. Buenos Aires: Ediciones Periferia, 1973.

WILDEN, Anthony. Sistemas y Estructuras. Buenos Aires: Alianza Editorial, 1979. 